\title{
Evaluation of APACHE II Score and QT Interval Dispersion for the Prognosis of Critical Cardiovascular Diseases
}

\author{
Chunhua MA ${ }^{1,2}$ \\ ${ }^{1}$ Department of Internal Medicine, Eastern Liaoning University, China \\ ${ }^{2}$ Department of Cardiology, Xuanwu Hospital, Capital Medical University, China, \\ *Correspondence to Chunhua MA, machunhua_0123456@163.com
}

Keywords: Critically ill cardiovascular disease; combined score; APACHE II score; QTd score.

ABSTRACT: Introduction: This pilot study evaluated the APACHE II Score and QT Interval Dispersion for the Prognosis of Critical Cardiovascular Diseases.

Materials and methods:A total of 217 Critically ill patients with cardiovascular disease were Selected in the Capital Medical University Xuanwu Hospital Cardiology CCU from January 2016 to January 2017. Patients were divided into death group and survival group according to the their survival situation. APACHE II Score and QT Interval Dispersion of the patients entering the CCU were recorded separately.

Results:The APACHE II score and QTd value in death group were significantly higher than those of survival group ( $<<0.05)$. The higher the APACHE II score and the QTd value, the higher the mortality rate, and the differences between the groups were statistically significant $(\mathrm{p}<0.05)$. There was a statistically significant difference between the combined score, the APACHE II score and the QTd score $(\mathrm{P}<0.05)$. AUC of the combined score was greater than APACHE II score.

Conclusion: The combined score, APACHE II score and QTd score were significantly higher in the death group. Combined score and APACHE II score were more accurate than QT dispersion to predict the mortality of critically ill patients with cardiovascular disease. The combined score was slightly higher than the APACHE II score to predict accuracy of critical cardiovascular disease. And APACHE II score in the specificity slightly stronger than the combined score.

\section{Introduction}

APACHE-II is currently the most widely used critically ill grading system in China. QTdispersion (QTd) is the degree of difference in QT interval between leads of 12-lead electrocardiogram, which reflects the heterogeneity of ventricular repolarization to a certain extent. And QTd on the evaluation of the prognosis of critically ill cardiovascular patients rarely involved. This study analyzed the value of APACHE II score and QTd score in the prognosis of critically ill patients with cardiovascular disease. We score APACHE II and QTd at the same time, and analyze the predictive level of the combined application.

\section{Materials and methods}

We selected 217 critically ill patients with cardiovascular disease in the Capital Medical University Xuanwu Hospital Cardiology Department of CCU from January 2016 to January 2017.All the patients will be divided into death group and survival group ccording to the survival condition.The APACHE II score an QTd score of the patients entering the CCU were recorded respectively.

\section{SAPS II scoring method}

The APACHE II score consists of 17 variables, including age, physiology variables (12 items), hospital type (emergency surgery, elective surgery, medical patients) and chronic diseases (including AIDS, metastatic cancer, hematological malignancies). Each minimum of 0 points, up to 26 points, a total score of 0-163 points.

\section{QT period dispersion}

We checked the conventional 12-lead ECG with a paper feed speed of $25 \mathrm{~mm} / \mathrm{s}$ and a calibration voltage of 1 $\mathrm{mV} / 10 \mathrm{~mm}$.QT interval measurement from the QRS integrated wave starting point to the T wave end point. The position of the T-wave end point was determined as the intersection of the $\mathrm{T}$ wave and the equipotential line. If 
there was an $\mathrm{u}$ wave, it was determined as the notch between the $\mathrm{T}$ wave and the $\mathrm{u}$ wave. Each lead to measure three, Whichever is the mean.QTd (QTd = QTmax- QTmin) is the difference which the longest QT interval minus the shortest QT interval.

\section{Statistical method}

SPSS16.0 software was used for statistical analysis. The measurement data is expressed as mean \pm standard deviation $(x \pm s)$, and the count data is expressed in terms of rate. The subjects' working curve and the area under the curve were plotted and calculated using the software. Comparison of area under the curve use Medcalc calculations by $\mathrm{Z}$ test. $\mathrm{P}<0.05$ for the difference was statistically significant.

\section{Results}

\section{Baseline characteristics of patients in the two groups}

There were 217 patients (Table 1), aged 50-46 (67 \pm 7.15$)$ years; male patients (139 cases) were more than female patients (78 cases).Patients with acute myocardial infarction (113 patients), heart failure (54 cases), unstable angina (36 cases), and other cardiovascular disease (14 patients). The APACHE II score and QTd value in the death group were significantly higher than those in the survival group. (Table 1).

Table1: Clinical characteristics of patients in survival and death Group

$\begin{array}{cllllll} & \mathrm{N} & \text { Sex (male/ female }) & \text { age } & \text { APACHEII } & \text { QTd }(\mathrm{ms}) \\ \text { All patients } & 217 & 139 / 78 & 67 \pm 7.15 & 35.73 \pm 11.57 & 101.29 & \text { 土 } \\ \text { survival } & 170 & 111 / 59 & 65 \pm 8.02 & 31.94 \pm 11.82 & 21.75 & 80.73 \pm 29.36 \\ \begin{array}{c}\text { group } \\ \text { death group }\end{array} & 47 & 28 / 19^{\mathrm{a}} & 69.4 \pm 7.63^{\mathrm{a}} & 48.69 \pm 11.24^{\mathrm{b}} & 152.11 & \text { 土 } \\ & & & & & 22.18^{\mathrm{b}}\end{array}$

a,compared with the survival group, $\mathrm{p}>0.05$; $\mathrm{b}$, compared with the survival group, $\mathrm{p}<0.05$.

Comparison of mortality rates in each scoring system segment

Mortality rate was the lowest $(0 \%)$ when APACHE II score $<25$ points and the highest $(64 \%)$ when APACHE II score $>46$ points. The higher the score, the higher the mortality rate. The differences among the different groups were statistically significant $(\mathrm{p}<0.05)$.

The mortality rate was 0 as APACHE II combined QTd score $\leq 26$. The mortality rate was $1.8 \%$ as combined score 26 to 30 points. The mortality rate of combined score 31 to 35 points was $5.6 \%$, mortality rate of combined score 36 to 40 points was $14.3 \%$, the mortality rate of combined score 41 to 45 points was $14.3 \%$ and the death rate of combined score $>46$ points was $78.3 \%$. As the scores were increased, the mortality rate was increased.The differences were statistically significant among the different groups $(\mathrm{p}<0.05)$.

Table2 : Comparison of mortality rates in each scoring system segment

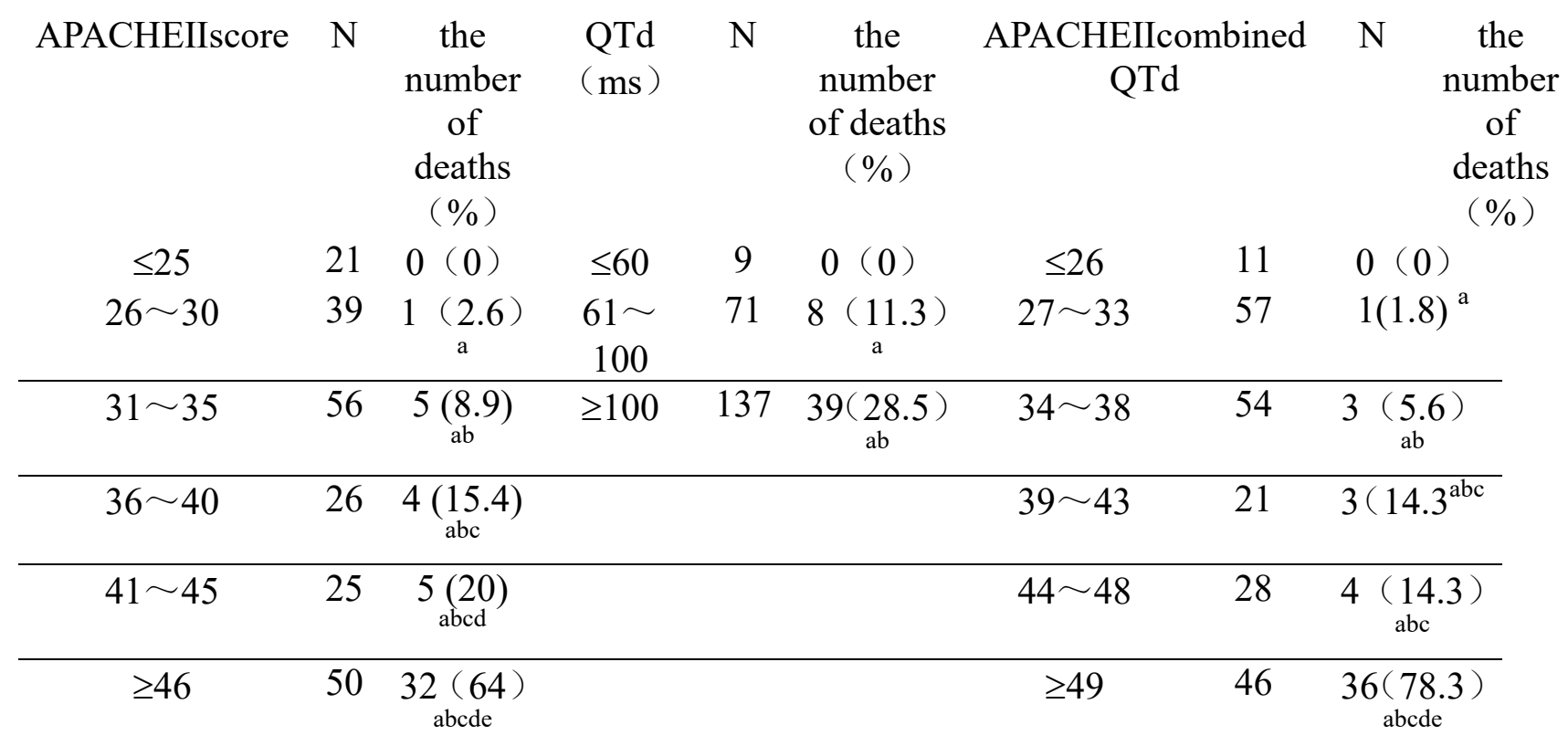

a, compared with the first group, $\mathrm{p}<0.05 ; \mathrm{b}$, compared with the second group, $\mathrm{p}<0.05$; c, compared with the third group, $\mathrm{p}<0.05$; $\mathrm{d}$, compared with the fourth group, $\mathrm{p}<0.05$; e, compared with the fifth group, $\mathrm{p}<0.05$.

ROC Curve analysis of Critical Cardiovascular Disease

AUC were calculated based on the APACHE II score, QTd and combined scores at different critical points for the sensitivity and specificity of the subject ROC.The results showed that the combined score of AUC was 0.931 , APACHE II score AUC was 0.814, QTd score AUC was 0.668 which could predict the probability of death in 
patients.

Comparing the area under the curve by the $\mathrm{Z}$ test showed that there was a statistically significant difference between the combined score , the APACHE II score and the QTd score, which was more accurate than the QTd score to predict the mortality of critically ill patients with cardiovascular disease.

There was no statistically significant difference between the combined score and the APACHE II score.But AUC of the combined score was greater than the APACHE II score that suggesting the combined score was significantly higher than the APACHE II score for critical cardiovascular disease predictors. And APACHE II score in the specificity slightly stronger than the combined score. (Table 3)(Figure 1).

Table 3 :The data of each scoring system for patient mortality prediction

\begin{tabular}{|c|c|c|c|c|}
\hline $\begin{array}{l}\text { Test result } \\
\text { ariable }\end{array}$ & AUC & Sensitivity & Specifici & $\begin{array}{l}95 \% \text { confiden } \\
\text { interval }\end{array}$ \\
\hline
\end{tabular}
APACHE II
0.814
0.802
0.921
$0.879-0.948$

$\begin{array}{lc}\text { QTd score } & 0.668 \mathrm{a} \\ \text { combined } & 0.931\end{array}$

0.815

0.473

$0.598-0.762$

score

0.877

0.863

a, compared with other groups, $\mathrm{p}<0.05$.

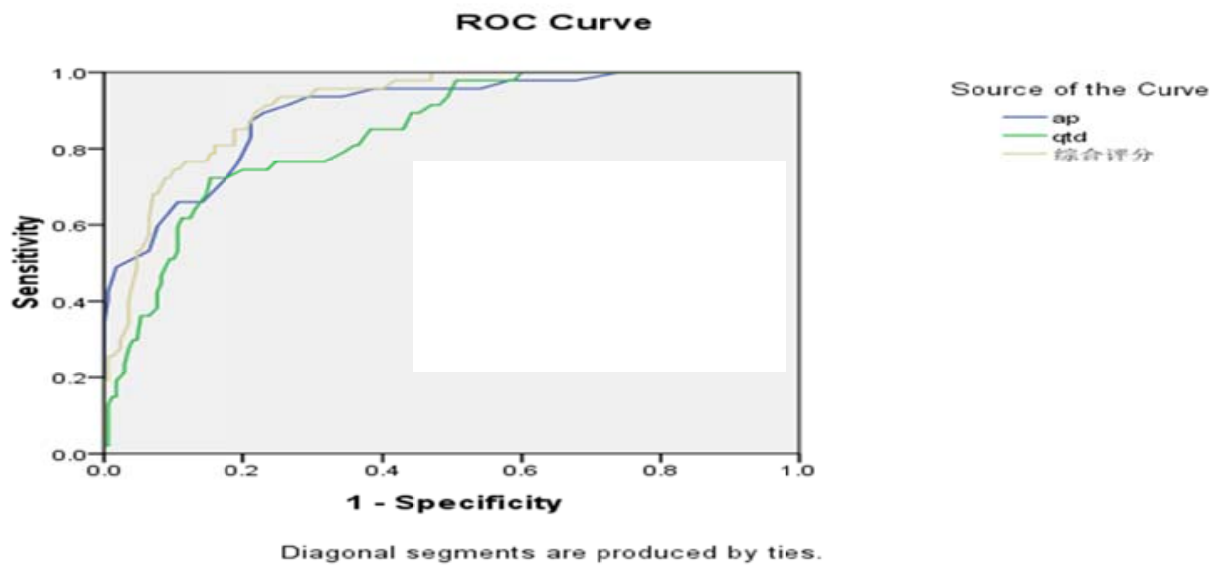

Figure 1: AUC of combined score, APACHE II score and QTd score

\section{Discussion}

The ideal forecasting system should be noninvasive, simple, accurate, and easy to collect data, and can be evaluated after diagnosis of the disease. Early assessment of course and prognosis are critical in predicting cardiovascular disease.Accurate prediction is conducive to timely and accurate intervention to improve the prognosis of patients. APACHE II scoring system can accurately predict the severity of severe disease early, mainly reflects the changes in systemic physiological indicators of patients, for local disease is not sufficient to reflect. QT interval dispersion through QTd describes the severity of cardiovascular disease, but not taking into account the situation of the whole body.There is no literature on the combination of the two reports, how to form a combination of clinical and effective in the formation of a complete scoring system, we need to conduct a deeper discussion. APACHE II scoring system can accurately predict the severity of severe disease early, mainly reflects the changes in systemic physiological indicators of patients, for local disease is not sufficient to reflect.And QT interval dispersion through QTd describes the severity of cardiovascular disease, but not taking into account the situation of the whole body.There is no literature on the combination APACHE II and QTd. We need to conduct a deeper discussion how to form a combination of clinical and effective in the formation of a complete scoring system.

The results of this study suggest that APACHE II score and QTd value in survival group were significantly lower than those in death group, indicating that APACHE II score and QT dispersion were significantly correlated to coronary heart disease prognosis. The APACHE II score was the higher, the mortality rate was the higher . The highest mortality rate (64\%) in the cardiovascular disease at APACHEIIscore $\geq 46$. The QTd value was the higher, the mortality rate was the higher.The highest mortality rate $(28.5 \%)$ was at QTd $\geq 100 \mathrm{~ms}$. The APACHE II combined QTd score was the higher, the mortality rate was the higher. The mortality rate was $78.3 \%$ at 
combined score $\geq 49$, significantly higher than the mortality at APACHE II score of 46 and QTd $\geq 100 \mathrm{~ms}$.It indicate that the combined score could better reflects the severity and prognosis for piatents with coronary heart disease.

ROC curve can be used to study the accuracy of clinical prediction system.By comparing AUC can fully reflect the diagnostic accuracy ,but not affected by the diagnostic threshold. From the analysis of this study can be seen the combined score were the highest in AUC, sensitivity and specificity for predicting the death of patients with critical cardiovascular disease compared to the APACHE II scoring system. The reason why the combined score increased the QT interval dispersion reflected in the cardiovascular local condition on the basis of APACHE II score.The above scenario shows that the combined score joints the advantages of APACHE II score and QT interval dispersion, which has been strengthened in predicting death.

The study collected 217 patients, comprehensive patient study data, collected a number of clinical indicators. The combined scoring system increased QT interval dispersion improved the predictive accuracy of critical cardiovascular disease to a certain extent by comparing the data among different groups while comparing AUC. The combined scoring system has a better advantage and provides an important reference for the individual treatment of patients. However, this study is a single center retrospective study, hoping to have a multi-center, large sample of joint research to further verify.

\section{References}

[1] Ohkubo K, Watanabe I, Okumura Y. Spatial and transmural repolarization, and dispersion of repolarization and late potentials evaluated using signal-averaged vector-projected 187-channel high-resolution electrocardiogram in Brugada syndrome. Journal of Arrhythmia 2013.

[2] Sauer AJ, Kaplan R, Xue J, et al. Electrocardiographic markers of repolarization heterogeneity during dofetilide or sotalol initiation for paroxysmal atrial fibrillation. Am J Cardiol 2014; 113:2030.

[3] Rubulis A, Bergfeldt L, Rydén L, Jensen J. Prediction of cardiovascular death and myocardial infarction by the QRS-T angle and $\mathrm{T}$ vector loop morphology after angioplasty in stable angina pectoris: an 8-year follow-up. J Electrocardiol 2010; 43:310. 